Research Article

\title{
A methanolic extract of Trigonella foenum-graecum (fenugreek) seeds regulates markers of macrophage polarization
}

\section{*Nurudeen Hassan, Cathryn Withycombe, Maninder Ahluwalia, Andrew Thomas, and Keith Morris}

Cardiff School of Health Sciences, Cardiff Metropolitan University, Western Avenue, Cardiff, CF5 2YB, United Kingdom

*Corresponding Author: Nurudeen Hassan, Cardiff School of Health Sciences, Cardiff Metropolitan University, Cardiff, CF5 2YB, United Kingdom

Submission Date: July 29, 2015, Acceptance date: December 24, 2015: Publication date: December 28, 2015

\begin{abstract}
Background: Macrophages are key cellular mediators in diabetes-related inflammation. Molecular cues such as cytokines found in the tissue microenvironment regulates the polarization of macrophages into an M1 (pro-inflammatory) or M2 (immunoregulatory) phenotype. Recent evidence suggests that M1 macrophages in diabetic patients may contribute to the complications associated with the disease such as atherosclerosis. Trigonella foenum- graecum (Tfg: fenugreek) seeds have been used in traditional medicine in Asia, Africa and the Middle-East for their alleged anti-diabetic properties.
\end{abstract}

Objective: To identify the molecular mechanism(s) through which $T f g$ seeds exert their effects, we investigated the role of a crude methanolic extract of $T f g$ seeds (FME) on macrophage polarization in vitro.

Method: THP-1 macrophages $(\mathrm{M} \phi)$ were treated with gBSA in the presence/absence of FME and the release and expression of M1 and M2 markers/cytokines were analysed. The role of FME on NF- $\mathrm{KB}$ activity was also explored using transfected HEK-293T cells.

Results: This study found that the $F M E$ significantly $(\mathrm{P}<0.05)$ decreased gBSA-induced secretion of M1 cytokines (TNF- $\alpha$, IL-1 $\beta$, IL-6 and IL-8) in THP-1 M $\phi$ cells. In the presence of gBSA, FME also significantly increased the gene expression of the M2 marker Dectin-1, but had no effect on IL-10, IL-1Ra. FME also significantly decreased TNF- $\alpha$ induced NF-kB reporter activity.

Conclusion: These results suggest that FME can regulate the expression of M1 and M2 markers in THP-1 M $\phi$ cells. This may be potentially through the modulation of NF-kB activity. Further work should be carried out to identify precise mechanism(s) involved in the effects of FME and Tfg seeds in diabetes and other conditions.

Keywords: chronic inflammation, macrophage polarization, diabetes, glycated BSA, THP-1 cells, Trigonella foenum graecum, fenugreek seeds, NF-кB, 


\section{INTRODUCTION}

Chronic low-grade inflammation is involved in the pathogenesis of obesity-related type-2diabetes and its complications[1]. Macrophages $(\mathrm{M} \phi)$ are key cellular mediators in diabetesrelated inflammation [2] and in response to different stimuli e.g. cytokines are polarized into classically activated (M1) or alternatively activated (M2) cells [3].

The inflammatory M1 sub-type is activated by IFN- $\gamma$ and bacterial lipopolysaccharide (LPS) and is characterised by the production of proinflammatory cytokines e.g. IL-1, IL-6 and TNF- $\alpha$. In contrast, the M2 macrophages are activated by IL- 4 and IL-13 and have a major role in immune regulation and tissue repair [4]. M2 macrophages produce cytokines and cell-surface receptors such as IL-10, IL-1 receptor antagonist (IL-1Ra) and Dectin-1, and down-regulate inflammatory processes associated with the M1 phenotype [5]. It is now understood that an imbalance in population of M1 and M2 and a predominance of M1 macrophages is a contributing factor to dysregulation of glucose metabolism in adipose tissue and leads to insulin resistance [6]. In line with this, studies have found that adipose tissue macrophages adopt an M1-like phenotype and produce large amounts of pro-inflammatory cytokines [4].A recent study also found that M2 macrophage polarization was associated with improved insulin resistance in diabetic mice [7].

Trigonella foenum graecum (Tfg) seeds are used in traditional Indian, Chinese and Middle-Eastern medicine for managing conditions including diabetes [8], metabolic disorder [9] and cancer [10]. More recently, Tfg seeds have been shown to decrease the release of proinflammatory cytokines such as TNF- $\alpha$ and Interleukin-1 $\beta$ in tissues of diabetic mice [11].

Neelakantan et al., conducted a meta-analysis on the effects of $T f g$ seeds on glycaemic control and found a strong association between $T f g$ intake and hypoglycaemic effect in diabetic patients [12]. In line with that, a saponin-rich water soluble extract of $T f g$; Fenfuro ${ }^{\mathrm{TM}}$ was recently patented in the US as an antidiabetic agent [13]. Taken together, the literature suggests a role for $T f g$ seeds in modulating diabetes and related inflammation.

In this study, we aimed to investigate the role of a methanolic extract of $T f g$ (FME) in regulating markers of M1 and M2 macrophages using an in vitro THP-1 M $\phi$ cell model.

\section{MATERIALS AND METHODS:}

Cell culture: Human promyelocytic cells (THP-1) were cultured in RPMI 1640 (Gibco, UK) supplemented with $10 \%$ heat inactivated Foetal Calf Serum; FCS (Labtech International), $1 \%$ sodium pyruvate, $1 \%$ non-essential amino acids and $1 \%$ penicillin $(100 \mathrm{IU} / \mathrm{ml})$, streptomycin $(100 \mu \mathrm{g} / \mathrm{ml})$ (Gibco, UK). For in vitro differentiation into macrophages, THP-1 monocytes $\left(6 \times 10^{5}\right.$ cells $\left./ \mathrm{ml}\right)$ were treated with $8 \mathrm{nM}$ phorbol-myristate-acetate; PMA (Sigma) for 48hours then maintained in fresh media without PMA for an additional 48hours before all experiments. In order to achieve a diabetes-related pro-inflammatory phenotype in THP-1 macrophages, cells were treated with $500 \mu \mathrm{g} / \mathrm{ml}$ of glycated-BSA (Sigma, UK). HEK293T (ATCC, Middlesex, UK) epithelial cells were grown in complete Dulbecco's Modified Eagle Medium (DMEM), 100U/ml penicillin $/ 100 \mu \mathrm{g} / \mathrm{ml}$ streptomycin mix and $4 \mathrm{mM}$ L-glutamine (Gibco, UK). 
Cell viability assay: Cells were cultured in a 96-well micro-titre plate for 48 hours. Varying concentrations of the FME was incubated with the cells for 24 hours and cell viability was determined using Cell Titer-Blue ${ }^{\circledR}$ (Promega) according to manufacturer's guidelines.

Extract preparation: $40 \mathrm{~g}$ of $T f g$ seeds were ground to fine powder and transferred into $100 \mathrm{ml}$ of $100 \%$ Methanol for incubation in a rotary mixer overnight at $25^{\circ} \mathrm{C}$. The solvent fraction was recovered by centrifugation $(6000 \mathrm{~g}$ for $15 \mathrm{mins})$ and the methanol evaporated in vacuo using a rotary evaporator (Bibby RE100, Staffordshire, UK). The residue was further subjected to $\mathrm{N}_{2}$ to obtain a dry residue. The residue (extract) was dissolved in dimethyl sulfoxide (DMSO) and reconstituted in culture media, filtered through a $0.22 \mu \mathrm{m}$ PVDF filter and stored at $-20^{\circ} \mathrm{C}$. For experiments, the extract was thawed and reconstituted in fresh media at the respective concentrations before adding to cells.

Quantification of Cytokines using ELISA: TNF- $\alpha$, IL-6 and IL-1 $\beta$ ELISA kits were obtained from R \& D systems (Abingdon, Oxford) and the assay undertaken using protocols outlined by manufacturer.

Quantitative reverse transcription-PCR (qRT-PCR): Total RNA was extracted from cell pellets using TRIzol ${ }^{\circledR}$ reagent (Life technologies). RNA was converted to cDNA using a High Capacity cDNA Reverse Transcription kit (Applied Biosystems, Warrington, UK). Gene expression was analysed by RT-PCR method using Fast SYBR® Green in combination with an Applied Biosystems Fast 7500 Real-Time PCR System (Applied Biosystems, Warrington UK). The comparative CT method $\left(2^{-\triangle \Delta C T}\right)$ was used to calculate relative gene expression. The primer sequences used were; GAPDH: 5'-CATTGACCTCAACTACATG-3' and 5'TCTCCATGGTGGTGAAGAC-3'; IL-10: 5'- ACGGCGCTGTCATCGATT- 3' and 5'TGGAGCTTATTAAAGGCATTCTTC- 3'; IL-1Ra: 5'-GGCCTCCGCAGTCACCTAATCAC- $\quad 3$ ' and 5'-GGACAGGCACATCTTCCCTCCAT-3', Dectin-1; 5'GGAAGCAACACATTGGAGAATGG -3' and 5'- CTTTGGTAGGAGTCACACTGTC -3'.

NF-kB reporter assay: HEK293T cells were grown in a 24-well plate to a confluence of 60$80 \%$. Cells were transfected with 50ng of NF-kB plasmid DNA and 5ng of the Renilla luciferase reporter DNA (Pr6tk; Promega, Southampton, UK) diluted in Opti-MEM® I Reduced-Serum Medium (Invitrogen Ltd, Paisley, UK) using Lipofectamine ${ }^{\circledR}$ transfection reagent (Invitrogen Ltd, Paisley, UK) for 4hours. The media was replaced with fresh media for another 20hours before experiments.

Statistical analysis: all data are presented as the mean \pm standard deviation. One-way analysis of Variance (ANOVA) was conducted for within group comparisons. The statistical package, Graphpad Prism 5 was used for all statistical analyses. Statistical significance is represented as: * $(\mathrm{P}$-value $<0.05)$; ** $(\mathrm{P}<0.01)$; *** $(\mathrm{P}<0.001)$. Error bars represent standard deviation of at least three replicates.

\section{RESULTS}

The effect of FME on THP-1 M $\phi$ cell viability. To determine the cytotoxic effect of FME on THP-1 M $\phi$, the cells were exposed to a range of concentrations $(30-460 \mu \mathrm{g} / \mathrm{ml})$ for 24 hours and then the viability of the cells was assessed. At the concentration of $120 \mu \mathrm{g} / \mathrm{ml}$ and above FME significantly reduced cell viability compared to untreated cells (Figure 1). 


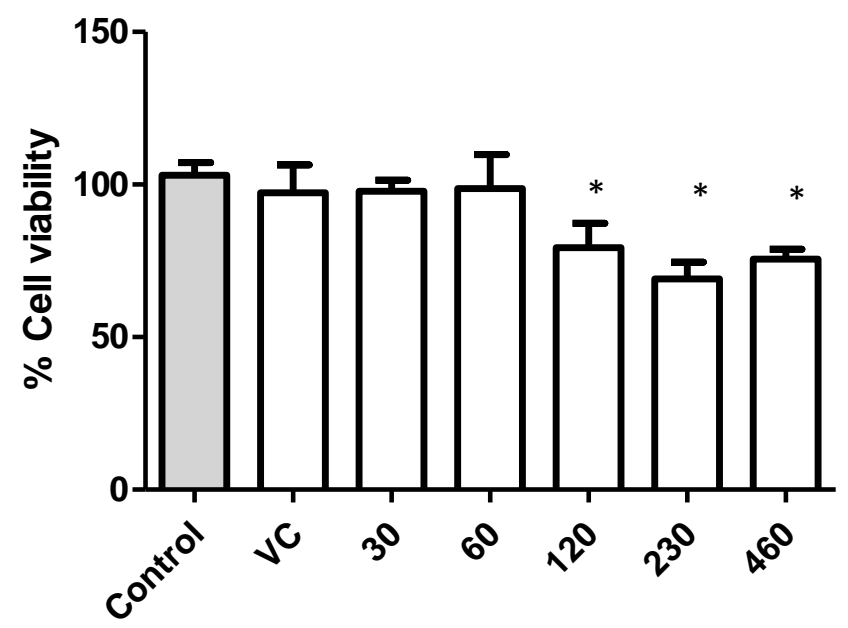

Figure 1. Effect of FME on cell viability. THP-1 M $\phi$ cells were treated with $30-460 \mu \mathrm{g} / \mathrm{ml}$ of FME and Vehicle Control (VC: DMSO 0.1\%) for 24 hours. The difference between control and treatment groups was determined by One-way ANOVA with Dunnett's post hoc test.

The effect of Fenugreek seed extract on gBSA induced TNF- $\alpha$ secretion in THP-1 M $\phi$ cells.

Using non-cytotoxic concentrations of FME, the effect of a range of concentrations 1$50 \mu \mathrm{g} / \mathrm{ml} \mathrm{FME}$ on gBSA-induced TNF- $\alpha$ release was studied in order to determine the optimal and range of bioactive concentrations. In the absence of gBSA, there was no significant increase in TNF- $\alpha$ secretion compared to the control and VC. All concentrations tested (1$50 \mu \mathrm{g} / \mathrm{ml}$ ) significantly reduced TNF- $\alpha$ secretion in the cells (Figure 2). The most potent concentration was $25 \mu \mathrm{g} / \mathrm{ml}$ FME, reducing g-BSA induced TNF- $\alpha$ secretion (1008.429 \pm $99.5 \mathrm{pg} / \mathrm{ml}$ vs. $532.1 \pm 94.2 \mathrm{pg} / \mathrm{ml})$. There was no significant difference in TNF- $\alpha$ levels in the treatment groups.

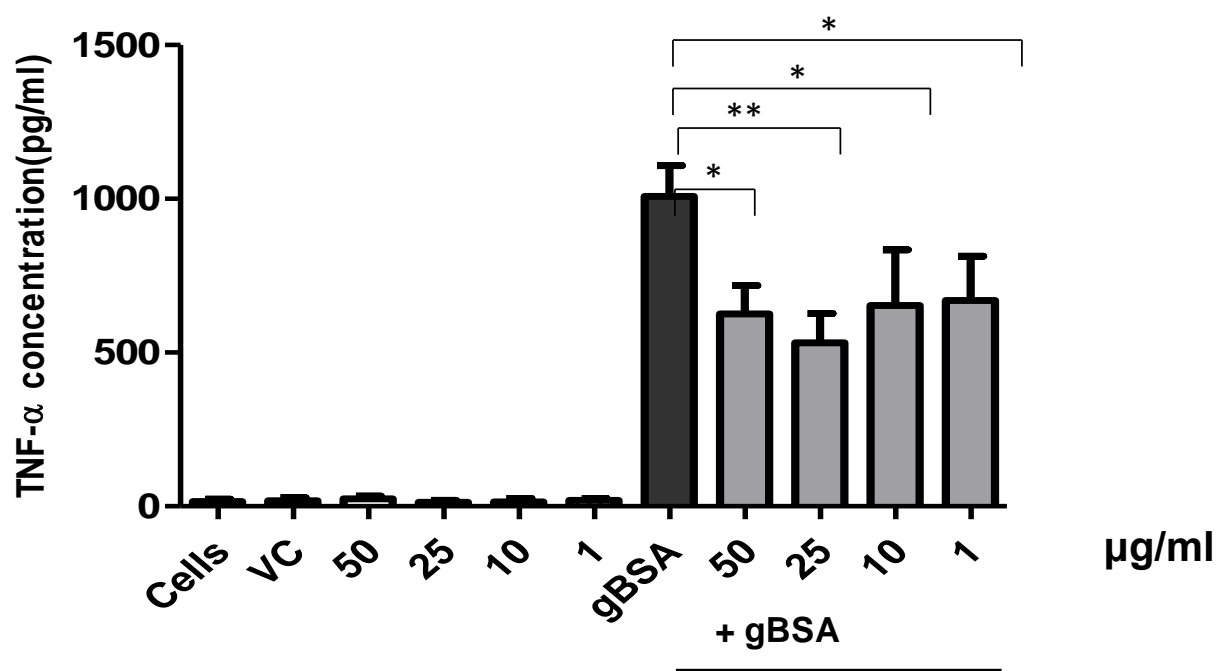

Figure 2. Effect of FME on gBSA-induced TNF- $\alpha$ release in THP-1 M $\phi$ cells. Cells were pre-treated with $1-50 \mu \mathrm{g} / \mathrm{ml} \mathrm{FME} \mathrm{for} 1$ hour and then the cells were treated with $500 \mu \mathrm{g} / \mathrm{ml}$ gBSA for 6 hours. The level of TNF- $\alpha$ secretion was analysed in the cell-free media using ELISA. The difference between all treatment groups was determined by One-way ANOVA with Tukey's post hoc test. 


\section{FME reduces the secretion of M1 cytokines in THP-1 M $\phi$ cells.}

It has been previously demonstrated that THP-1 macrophages express a number of M1(proinflammatory) cytokines such as TNF- $\alpha$, IL-1 $\beta$ and IL-6 upon stimulation of by proinflammatory stimuli such as bacterial LPS and Interferon- $\gamma$ (IFN- $\gamma$ ) [14]. Upon activation, M1 macrophages are characterised by the ability to secrete high levels of TNF- $\alpha$, IL1- $\beta$ and IL-6 cytokines [15]. Having demonstrated the effect of FME on TNF- $\alpha$ secretion in the presence of gBSA (figure 2), we further investigated the role of FME in regulating the secretory pattern of a panel of M1 cytokines within an inflammatory milieu at an early and later time point. At a concentration of $29 \mu \mathrm{g} / \mathrm{ml}$, we found that FME was able to significantly reduce gBSA induced levels of TNF- $\alpha$ (figure 3), IL-1 $\beta$ (figure 4) and IL-6 (figure 5) at 6 and 24hours.
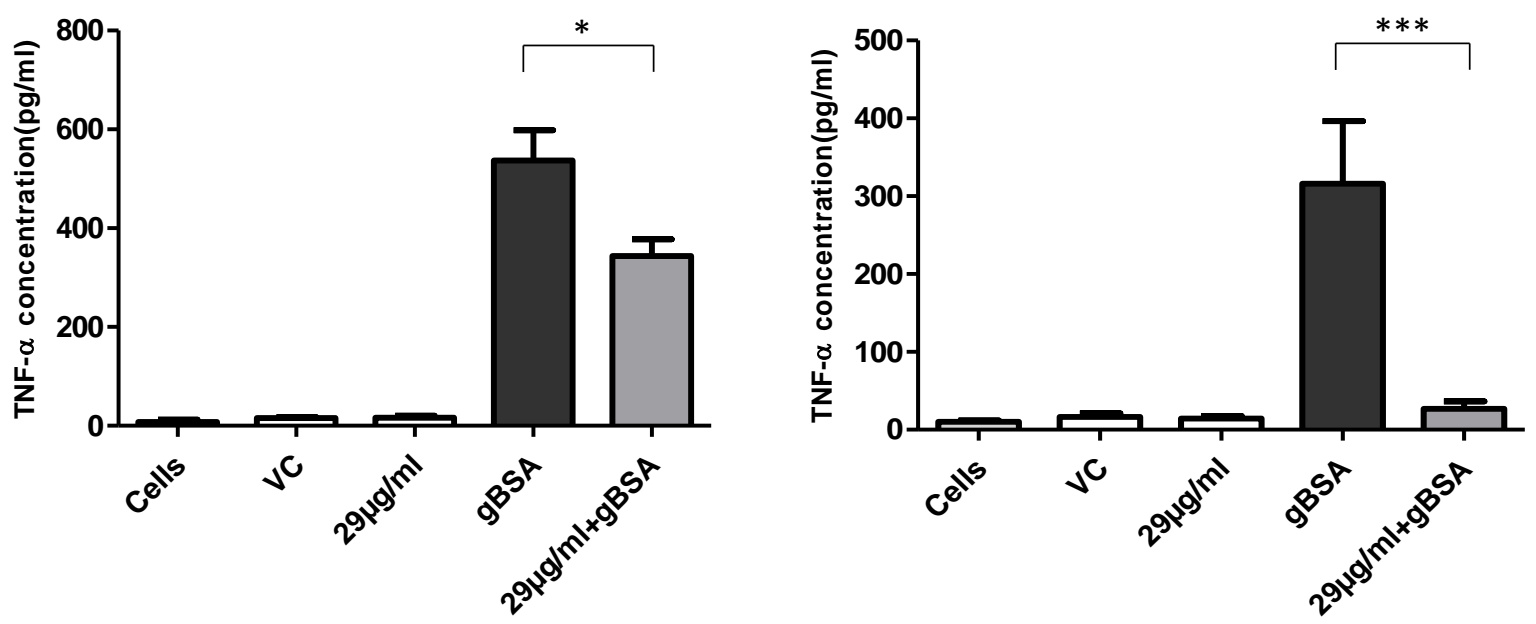

Figure 3. Effect of FME on gBSA-induced TNF- $\alpha$ release in THP-1 M $\phi$ cells. Cells were pre-treated with $29 \mu \mathrm{g} / \mathrm{ml} \mathrm{FME} \mathrm{for} 1$ hour and then the cells were treated with $500 \mu \mathrm{g} / \mathrm{ml}$ gBSA for a) 6 hours and b) 24 hours. The level of TNF- $\alpha$ secretion was analysed in the cellfree media using ELISA. The difference between treatment groups was determined by Oneway ANOVA with Tukey's post hoc test.
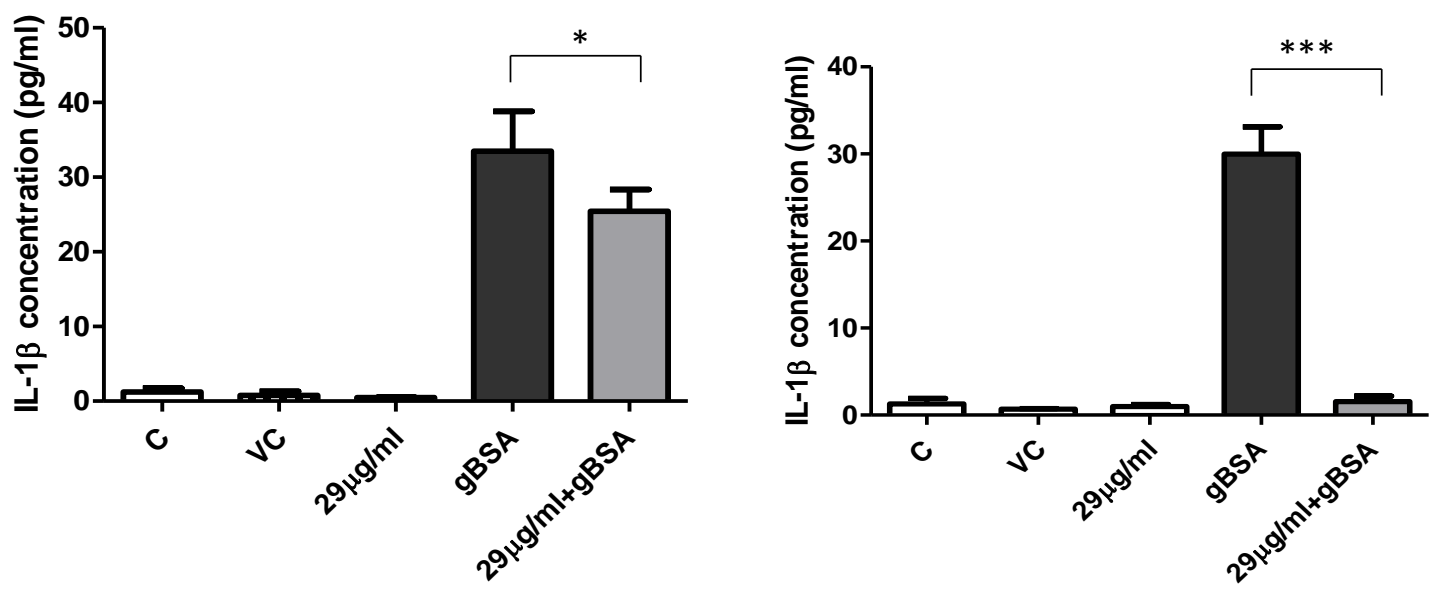

Figure 4. Effect of FME on gBSA-induced IL-1 $\beta$ release in THP-1 M $\phi$ cells. Cells were pretreated with $29 \mu \mathrm{g} / \mathrm{ml} \mathrm{FME}$ for 1 hour and then the cells were treated with $500 \mu \mathrm{g} / \mathrm{ml} \mathrm{gBSA}$ for a) 6 hours and b) 24 hours. The level of IL- $1 \beta$ secretion was analysed in the cell-free media using ELISA. The difference between treatment groups was determined by One-way ANOVA with Tukey's multiple comparison post hoc test. 
a

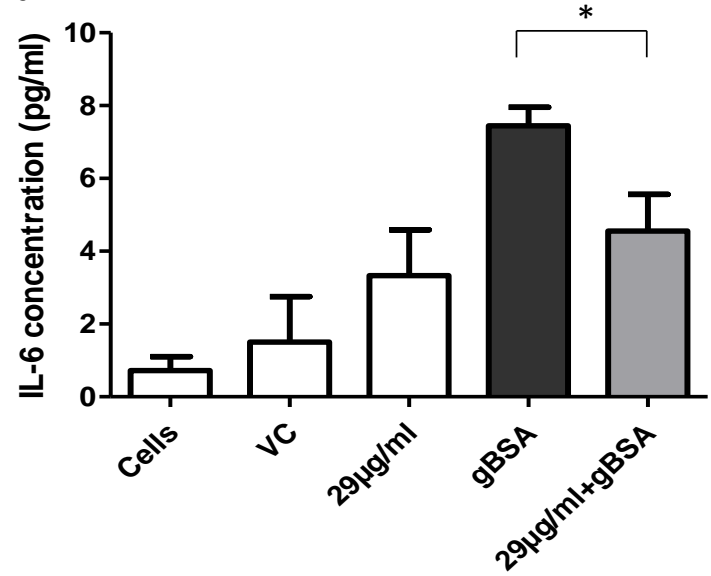

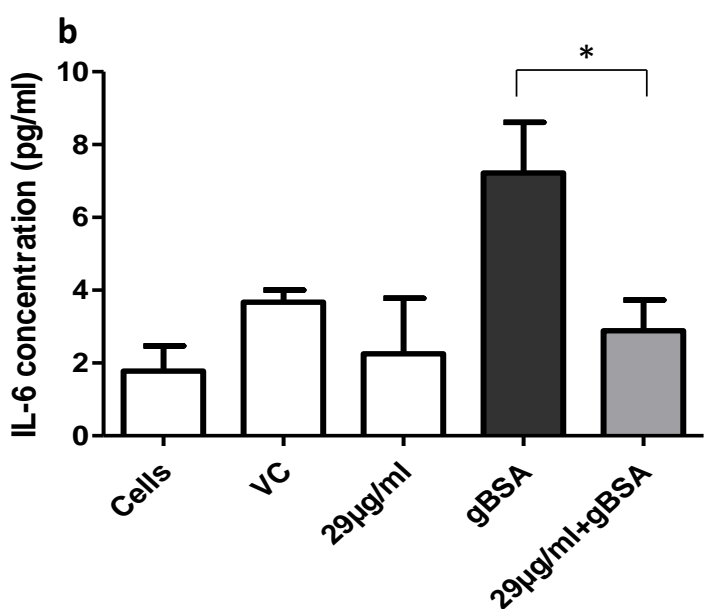

Figure 5. Effect of FME on gBSA-induced IL-6 release in THP-1 M $\phi$ cells. Cells were pretreated with $29 \mu \mathrm{g} / \mathrm{ml} \mathrm{FME}$ for 1 hour and then the cells were treated with $500 \mu \mathrm{g} / \mathrm{ml} \mathrm{gBSA}$ for a) 6 hours and b) 24 hours. The level of IL- 6 secretion was analysed in the cell-free media using ELISA. The difference between treatment groups was determined by One-way ANOVA with Tukey's multiple comparison post hoc test.

\section{The effect of FME on M2 markers in THP-1 macrophages}

After demonstrating that FME reduces the secretion of M1 cytokines (figure 3-5), we further investigated whether FME up-regulates the expression of M2 markers and can favour an M2 phenotype in the presence of pro-inflammatory gBSA. THP- $1 \mathrm{M} \phi$ cells were pre-treated with FME and then stimulated with gBSA and the expression of M2 genes was analysed using qRT-PCR. There was a significant increase in the gene-expression of IL-10 and IL-1Ra (figure $6 \mathrm{a} \& 6 \mathrm{~b}$ ) in cells treated with gBSA alone and a combination of FME and gBSA, however, there was no significant difference between gBSA and FME-gBSA. Dectin-1 demonstrated a different expression pattern; in the presence of FME-gBSA, there was a significant increase in Dectin-1 expression at mRNA level (figure 6c).

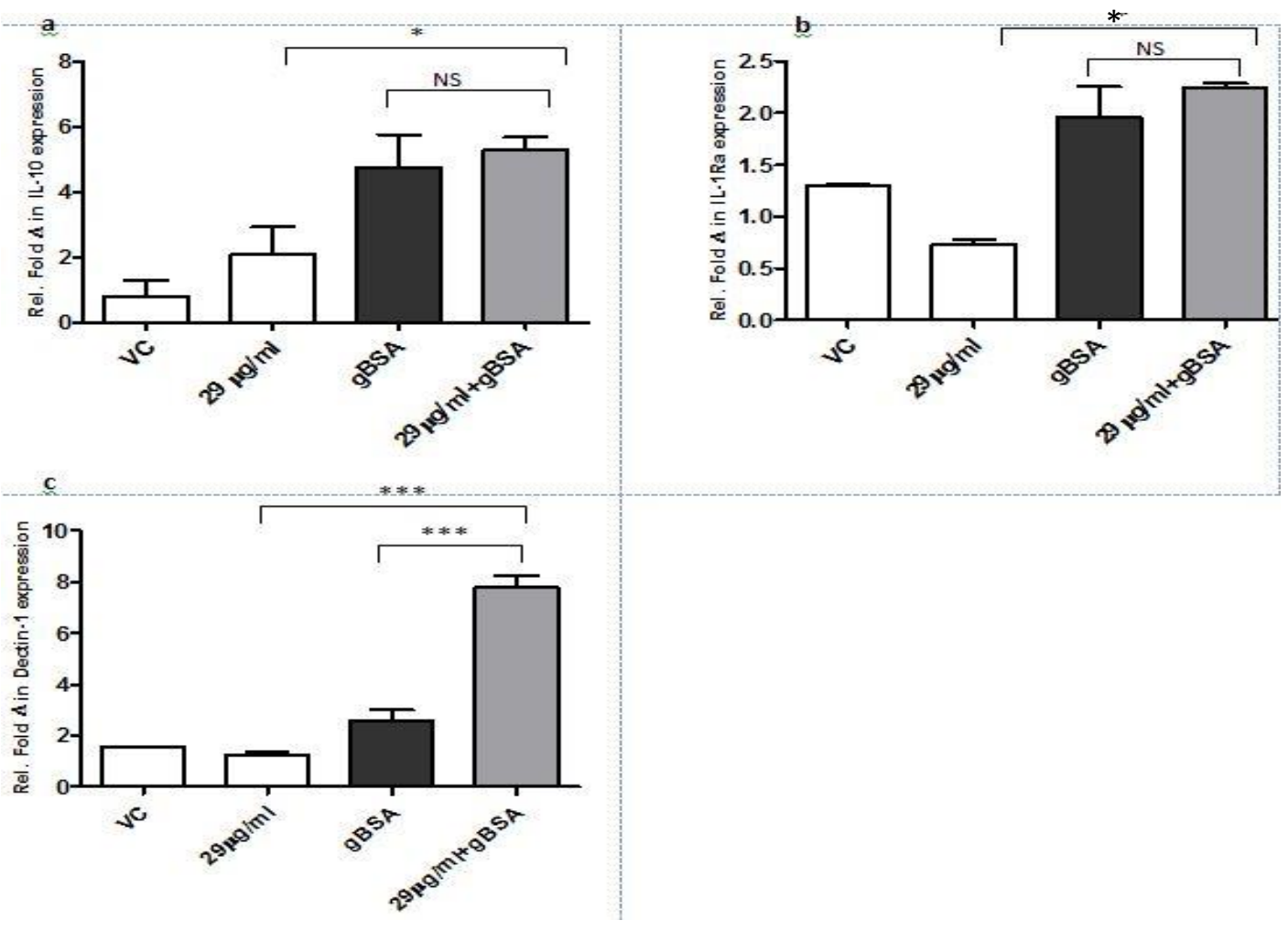


Figure 6. Effect of FME on M2 markers: IL-10, IL-1Ra and Dectin-1 expression in THP-1 M $\phi$ cells. Cells were pre-treated with $29 \mu \mathrm{g} / \mathrm{ml} \mathrm{FME}$ for 1 hour and then the cells were treated with $500 \mu \mathrm{g} / \mathrm{ml}$ gBSA for 24 hours. The RNA from the cell pellets was collected and the expression of M2 makers a) IL-10, b) IL-1Ra, and c) Dectin-1 was analysed using qRT-PCR. The difference between treatment groups was determined by One-way ANOVA with Tukey's multiple comparison post hoc test. NS: not significant.

\section{FME significantly reduces TNF-alpha induced NF-kB reporter activity.}

To determine the mechanism through which the observed effects FME may be mediated, NF$\kappa \mathrm{B}$ gene reporter assay was carried out. Due to the fact that FME reduced TNF- $\alpha$ secretion (fig 2-3) and TNF- $\alpha$ is a known target gene of NF-kB [16], the effect of FME on NF-kB activity was explored as a potential target for the anti-inflammatory activity of the extract. Many nutraceutical agents are known to exert anti-inflammatory effects through inhibition of NF-kB signalling [17]. In the presence of the TNF- $\alpha$, there was a significant increase in NF$\mathrm{kB}$ reporter activity which was significantly reduced by pre-treatment with FME (figure 7).

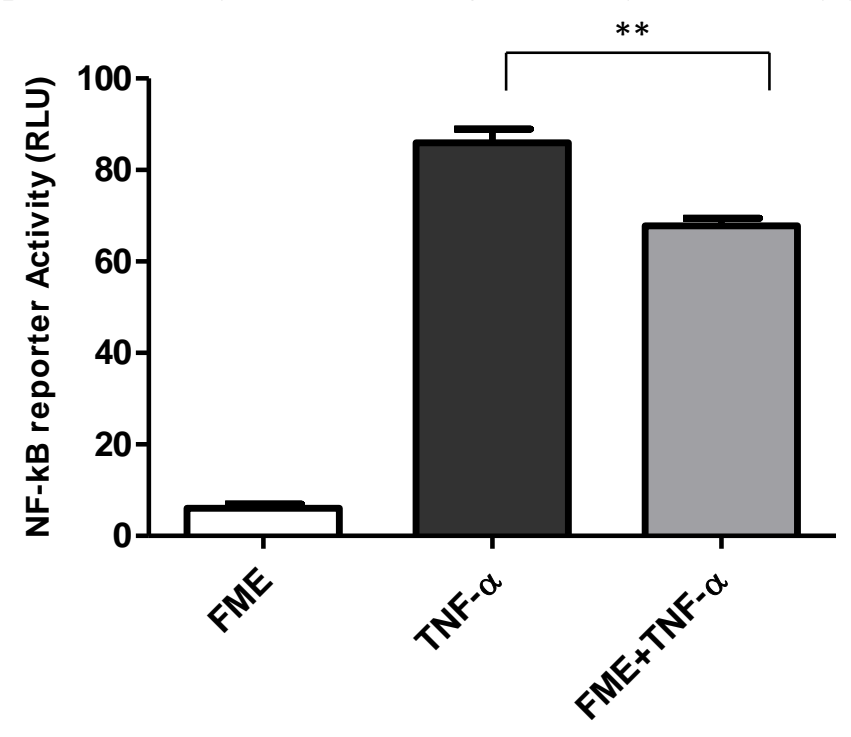

Figure 7. FME significantly attenuates TNF-alpha stimulated NF-kB activity. HEK-293T cells were co-transfected with NF-kB and Renilla control plasmids for $24 \mathrm{hr}$. The cells were then pre-treated with FME for 1hour. The cells were then stimulated with 10ng/ml TNFalpha. After 24hours post-stimulation, the cells were lysed and gene-reporter activity was analysed using the dual-glo luciferase assay.

\section{DISCUSSION}

The role of macrophage polarisation in obesity-linked metabolic disorders such Type-2diabetes is currently an area that is being extensively explored. The state of chronic lowgrade inflammation as found in obesity can lead to polarization of macrophages from an immune-regulatory M2 to a proinflammatory M1 phenotype [18].

In this study, the role of a methanolic extract of $T f g$ seeds on macrophage polarization was explored using the human THP-1 macrophage cells. Previous studies have demonstrated that THP-1 macrophages can be polarized into M1 and subtypes of M2 macrophages (M2a and M2c) in vitro [19] and hence was an appropriate model for a study whose primary aim was the regulation of polarization of these immune cells. For instance, in a recent paper, 
Chanput and colleagues explored the polarizing ability of LPS and food compounds such as vitamin D3 and Lentinan in THP-1 macrophages [14]. Although their study found that the food components (Vitamin D3 and Lentinan) had no effect on macrophage polarization, in contrast another paper by Gao et al. reported an M2 phenotype switch in macrophages mediated through release of IL-4 and/or IL-13 following treatment with Curcumin, a widely used food product [20].

$T f g$ seeds have been associated with many beneficial effects including antidiabetic, antibacterial, hypocholesterolaemic, anti-inflammatory, anticarcinogenic and hepatoprotective (see review [21]). We hypothesised that the reported clinical effects of Tfg may be in part attributable to the role of its bioactive flavonoid compounds in attenuating diabetes-related inflammatory processes such as M1 macrophage polarization. In our study we demonstrated that FME significantly reduced gBSA induced secretion of TNF- $\alpha$, IL-1 $\beta$ and IL- 6 by THP-1 macrophages.

Chemical analysis of a hydromethanolic extract of $T f g$ seeds by Benayad et al., found that the main compounds present were flavonoid glycosides; mainly, apigenin and luteolin glycosides [22]. In concordance with their study, we found that chemical analysis of FME exhibited a similar profile of chemical compounds (data unpublished).

Along with a reduction in M1 cytokines, FME also induced a significant upregulation in the gene expression of an M2 marker; Dectin-1, in the presence of gBSA (fig. 6). There was no difference in IL-10 and IL-1Ra expression compared to the gBSA treatment.

Although inconclusive, our results suggest that FME has the potential to induce a shift towards an M2 phenotype that is characterised by a high Dectin-1 and low IL-10 and IL-1Ra expression. Dectin-1 is a marker that is associated with the M2b subtype of macrophages [23]. Consequently, we propose that other M2b signature markers such as MR and SIGNR3 should be studied to confirm if FME can specifically induce M2b-like phenotype.

Our work also revealed that FME attenuates TNF- $\alpha$ induced NF- $\kappa B$ gene reporter activity. NF- $\kappa \mathrm{B}$ plays a central role in the signalling of gBSA (an advanced glycation end product; AGE) [24] and M1 macrophage polarization [25]. Upon binding of AGEs to the receptor for AGE (RAGE), the transcription factor NF-kB is activated, initiating a cellular signalling cascade that results in the production of proinflammatory cytokines such as TNF- $\alpha$, and IL-1 and IL-6 [26]. The ability of FME to reduce NF- $\kappa$ B activity in the presence of a proinflammatory cytokine (TNF- $\alpha$ ) suggests that FME may exert M $\phi$ polarization through modulating the activity of cellular and transcription factors.

There are a number of other molecular and cellular mechanisms that play a major role in $\mathrm{M} \phi$ polarization. This study only explored a select group of cytokines, cellular receptors and transcription factor involved in this process. Other major molecular mechanisms involved in M1-M2 switch include Nod-like receptors [27], cytokine signal inhibitors (suppressor of cytokine signalling; SOCS [28]) and transcription factors such as Peroxisome ProliferatorActivated Receptors (PPARs), Activated Protein-1 (AP-1), Glucocorticoid Receptors, IFN regulatory factors (IRFs), Signal Transducers and Activators of Transcription (STATs) and Krüppel-Like Factors (KLFs) (see review [25]). Due to the regulatory role of transcription factors in M1-M2 polarization in macrophages and disease, the effect of FME on these transcription factors should be explored.

Conclusion: The current work has demonstrated that the methanolic extract of Tfg seeds exert potent anti-inflammatory effects and can modulate the release and expression of M1 
and M2 markers in a human in vitro macrophage model. These effects appear to be mediated through the regulation of the transcription factor NF-kB, however, further work needs to be carried out to confirm the exact mechanisms through which these effects are mediated and whether these effects would also be observed in vivo.

Abbreviations: Tfg (Trigonella foenum-graecum); FME (methanolic extract of $T f g$ ); TNF- $\alpha$ (tumour necrosis factor- $\alpha$ ); IL-1 $\beta$ (Interleukin-1 $\beta$ ); NF- $\kappa \mathrm{B}$ (Nuclear factor- $\kappa \mathrm{B}$ ); IL-10 (Interleukin-10); IL-1Ra (Interleukin-1 Receptor Antagonist); qRT-PCR (quantitative-reverse transcription-polymerase chain reaction).

Competing Interests: The authors declare that they have no conflicts of interest.

Author's contributions: All authors contributed to this study.

Acknowledgments: We would like to thank Khalid Al Matari, Sumaiya As Siyabi, Salim Al Musafri, Zulaikha Al-Harthi and Salima Sultan for their technical assistance. This work was supported by Dr Christoph and Pauline Hering, Switzerland.

\section{REFERENCES}

1. Weber, K.S., et al., The Role of Markers of Low-Grade Inflammation for the Early Time Course of Glycemic Control, Glucose Disappearance Rate, and beta-Cell Function in Recently Diagnosed Type 1 and Type 2 Diabetes. Diabetes Care, 2015. 7.

2. Chawla, A., K.D. Nguyen, and Y.P. Goh, Macrophage-mediated inflammation in metabolic disease. Nat Rev Immunol, 2011. 11(11): p. 738-49.

3. Mantovani, A., A. Sica, and M. Locati, New vistas on macrophage differentiation and activation. Eur J Immunol, 2007. 37(1): p. 14-6.

4. Zeyda, M., et al., Human adipose tissue macrophages are of an anti-inflammatory phenotype but capable of excessive pro-inflammatory mediator production. Int $J$ Obes, 2007. 31(9): p. 1420-8.

5. Zeyda, M. and T.M. Stulnig, Adipose tissue macrophages. Immunology Letters, 2007. 112(2): p. 61-67.

6. Kraakman, M.J., et al., Macrophage polarization in obesity and type 2 diabetes: weighing down our understanding of macrophage function? Front Immunol, 2014. 5(470).

7. Hussaarts, L., et al., Chronic helminth infection and helminth-derived egg antigens promote adipose tissue M2 macrophages and improve insulin sensitivity in obese mice. Faseb J, 2015. 29(7): p. 3027-39.

8. Medagama, A.B. and R. Bandara, The use of complementary and alternative medicines (CAMs) in the treatment of diabetes mellitus: is continued use safe and effective? Nutr J, 2014. 13: p. 102.

9. Hassanzadeh Bashtian, M., et al., Evaluation of Fenugreek (Trigonella foenum-graceum L.), Effects Seeds Extract on Insulin Resistance in Women with Polycystic Ovarian Syndrome. Iran J Pharm Res, 2013. 12(2): p. 475-81.

10. Alsemari, A., et al., The selective cytotoxic anti-cancer properties and proteomic analysis of Trigonella Foenum-Graecum. BMC Complement Altern Med, 2014. 14: p. 114. 
11. Gupta, S.K., et al., Effects of Trigonella foenum-graecum (L.) on retinal oxidative stress, and proinflammatory and angiogenic molecular biomarkers in streptozotocininduced diabetic rats. Mol Cell Biochem, 2014. 388(1-2): p. 1-9.

12. Neelakantan, N., et al., Effect of fenugreek (Trigonella foenum-graecum L.) intake on glycemia: a meta-analysis of clinical trials. Nutr J, 2014. 13(7): p. 1475-2891.

13. Swaroop, A., et al., Safety, efficacy and toxicological evaluation of a novel, patented anti-diabetic extract of Trigonella Foenum-Graecum seed extract (Fenfuro). Toxicol Mech Methods, 2014. 24(7): p. 495-503.

14. Chanput, W., et al., Characterization of polarized THP-1 macrophages and polarizing ability of LPS and food compounds. Food Funct, 2013. 4(2): p. 266-76.

15. Chavez-Galan, L., et al., Much More than M1 and M2 Macrophages, There are also CD169(+) and TCR(+) Macrophages. Front Immunol, 2015. 6(263).

16. Lee, B.C. and J. Lee, Cellular and molecular players in adipose tissue inflammation in the development of obesity-induced insulin resistance. Biochim Biophys Acta, 2014. 3: p. 446-62.

17. Aggarwal, B.B., et al., Molecular targets of nutraceuticals derived from dietary spices: potential role in suppression of inflammation and tumorigenesis. Exp Biol Med, 2009. 234(8): p. 825-49.

18. Lumeng, C.N., J.L. Bodzin, and A.R. Saltiel, Obesity induces a phenotypic switch in adipose tissue macrophage polarization. J Clin Invest, 2007. 117(1): p. 175-84.

19. Spencer, M., et al., Adipose tissue macrophages in insulin-resistant subjects are associated with collagen VI and fibrosis and demonstrate alternative activation. Am J Physiol Endocrinol Metab, 2010. 299(6): p. 14.

20. Gao, S., et al., Curcumin induces M2 macrophage polarization by secretion IL-4 and/or IL-13. J Mol Cell Cardiol, 2015. 85: p. 131-139.

21. Yadav, U.C. and N.Z. Baquer, Pharmacological effects of Trigonella foenumgraecum L. in health and disease. Pharm Biol, 2014. 52(2): p. 243-54.

22. Benayad, Z., C. Gomez-Cordoves, and N.E. Es-Safi, Characterization of flavonoid glycosides from fenugreek (Trigonella foenum-graecum) crude seeds by HPLC-DADESI/MS analysis. Int J Mol Sci, 2014. 15(11): p. 20668-85.

23. Lefevre, L., et al., The C-type lectin receptors dectin-1, MR, and SIGNR3 contribute both positively and negatively to the macrophage response to Leishmania infantum. Immunity, 2013. 38(5): p. 1038-49.

24. Anisuzzaman, et al., Longistatin in tick saliva blocks advanced glycation end-product receptor activation. J Clin Invest, 2014. 124(10): p. 4429-44.

25. Tugal, D., X. Liao, and M.K. Jain, Transcriptional control of macrophage polarization. Arterioscler Thromb Vasc Biol, 2013. 33(6): p. 1135-44.

26. Singh, R., et al., Advanced glycation end-products: a review. Diabetologia, 2001. 44(2): p. 129-46.

27. Vandanmagsar, B., et al., The NLRP3 inflammasome instigates obesity-induced inflammation and insulin resistance. Nat Med, 2011. 17(2): p. 179-88.

28. Wilson, H.M., SOCS Proteins in Macrophage Polarization and Function. Front Immunol, 2014. 5(357). 